\title{
Brief School Performance Guide with Students with Autism Spectrum Disorders
}

\author{
Manuel Ojea Rua* \\ Univeristy of Vigo, Spain
}

*Corresponding author: Manuel Ojea Rua, Univeristy of Vigo, Spain.

Received Date: February 14, 2019

Published Date: February 22, 2019

\section{Opinion}

When a student with Autism Spectrum Disorder (ASD) arrives at school great worry may arise in educational community, whose first reaction could be evading that responsibility and refer it to centre specialist services for differentiated attention. However, we must be indicating some general priority advice, although each situation is particular, which can help to face an effective social and educational inclusion in different levels of the intervention: 1) in center level, 2) in classroom context, 3) over individual aspect and 4) in specific aspects [1].

\section{In Center Level}

From perspective of center collegiate action, it's necessary to elaborate an action basic protocol, which includes following aims:

a. Evaluate initial situation with collaboration of family and other participating social resources.

b. Delimit and request training based on needs itself, related to situation demand.

c. Specify and request complementary support resources adjusted to this initial evaluation.

d. Design and structure from an inclusive perspective the performance of support resources, support type, support modality, spaces and times (...)

e. Promote creation of specific commissions in center to specify the adapted educational measures.

f. Appoint a specific tutor teacher of reference in center.

g. Appoint a reference tutor student in classroom of individual with ASD.

h. Create a team of older students and support level for nonformal spaces.

i. Facilitate coordination of family and social services with different commissions and tutors, from initial evaluation processes to design of educational action and its evaluation follow- up.

j. Ensure compliance with quality criteria and coexistence in center previously agreed.

\section{In Classroom Context}

In the class, it's necessary to make following adaptations:

\section{I) in spatial location:}

a. Place it'll occupy in the different classrooms must be clearly indicated.

b. Signal the indicated places.

c. Space reserved located frontally towards next to digital screen.

d. Space must be away from windows, doors and corridors that can act as distracting stimuli of attention.

e. Reduce noise, e.g., changes class bell can replace by a light signal.

f. Avoid strong smell, derived from perfumes (...)

II) In curricular structure:

a. Anticipate and present the objectives clearly.

b. Present a single content on each occasion.

c. Specify various materials intended to acquire such content.

d. Verify and corroborate that said content has been acquired before accessing following content.

e. Facilitate the social reinforcement before the accomplishment of the learning be correct or not.

f. Make the execution time of proposed tasks more flexible. 
g. Make the exam time more flexible, giving the necessary time for its development.

h. Adapt the exam way, being preferable tests based on short answers or type-test.

i. Ask questions clearly avoiding elements that include more than one concept, as may give rise to doubts.

j. Avoid questions that require relationships or deductions, except if it have been previously tested.

k. Ensure the student have understood what is the question.

\section{III) In organizational level:}

a. Facilitate work in peer groups, with collaboration of reference student-tutor.

b. Facilitate cooperative work of groups of 4-5 students, which is maintained permanently throughout the course.

c. If it's necessary, encourage also individual work over regular context.

d. If it's necessary, delimit those educational measures adapted from educational reinforcement or curricular adaptation.

e. If required, design the structure of related support process.

\section{At Individual Level}

Our relationship with a person with ASD must take into account following advice:

a. Speak him looking into his eyes, in a calm, calm tone, avoiding changes of tone and with a clear and direct language that avoids turns, metaphors or misinterpretations, e. g, not to say: "we stayed at door (...)", otherwise, "we stayed at class door at break (...)"

b. Call him by his name, touching him lightly.

c. Indicate that we have understood what you are trying to tell us, even if you have spoken between lines. d. Allow time for you to organize your thoughts and understand the comments or demands of context.

e. Don't make fun of your comments and be patient if you contextualize the conversation at a certain moment.

f. Recognize use of a multi-personal agenda of several categories, with visual warning alarm, which signals start of the planned activities.

g. Fulfil with temporary structure of planned activities and if they occur alteration or unforeseen events, explain and anticipate the new situation and write it down in the personal agenda.

\section{At Specific Level}

Due to its specific characteristics, is required to encourage development of following specific aims:

A. Facilitate interaction and social communication through a formal structure based on group learning with the collaboration of specialist teaching.

B. Ease development of interaction and social communication in informal activities, with collaboration- mediation of students support team.

C. Design the behavioral interventions, which may be necessary, with collaboration of support specialist teaching, the family and health and social services, in order to avoid measures, treatments and / or contrary actions between themselves.

\section{Acknowledgement}

None.

\section{Conflict of Interest}

Author declare no conflict of interest.

\section{References}

1. Ojea M (2018) What to do when a student with Autism Spectrum Disorder arrived to our educational center? Brief action guide. In press. 